\title{
EXACT NULL CONTROLLABILITY, COMPLETE STABILIZABILITY AND CONTINUOUS FINAL OBSERVABILITY OF NEUTRAL TYPE SYSTEMS
}

\author{
$\operatorname{RABAH~RABAH}^{a, *}$, GRIGORY SKLYAR ${ }^{b}$, PAVEL BARKHAYEV $^{c}$ \\ ${ }^{a}$ Research Institute of Communication and Cybernetics \\ IMT Atlantique, Mines-Nantes, 4 rue Alfred Kastler, BP 20722, 44307 Nantes, France \\ e-mail: rabah@emn.fr \\ ${ }^{b}$ Institute of Mathematics \\ University of Szczecin, Wielkopolska 15, 70-451 Szczecin, Poland \\ e-mail: sklar@univ.szczecin.pl \\ ${ }^{c}$ Institute for Low Temperature Physics and Engineering \\ National Academy of Sciences, 47 Lenin Ave., 61103 Kharkiv, Ukraine \\ e-mail: barkhayeveilt.kharkov.ua
}

\begin{abstract}
For abstract linear systems in Hilbert spaces we revisit the problems of exact controllability and complete stabilizability (stabilizability with an arbitrary decay rate), the latter property being related to exact null controllability. We also consider the case when the feedback is not bounded. We obtain a characterization of complete stabilizability for neutral type systems. Conditions for exact null controllability of neutral type systems are discussed. By duality, we obtain a result about continuous final observability. Illustrative examples are given.
\end{abstract}

Keywords: exact null controllability, complete stabilizability, final observability, neutral type system.

\section{Introduction}

Consider the controlled neutral type system

$$
\dot{z}(t)=A_{-1} \dot{z}(t-1)+L z_{t}(\cdot)+B u(t),
$$

where

$$
L z_{t}(\cdot)=\int_{-1}^{0}\left[A_{2}(\theta) \dot{z}(t+\theta)+A_{3}(\theta) z(t+\theta)\right] \mathrm{d} \theta,
$$

with $z(t) \in \mathbb{R}^{n}, u(t) \in \mathbb{R}^{m}$, and the matrices $A_{-1}, A_{2}$, $A_{3}$ and $B$ are of appropriate dimensions. The elements of $A_{2}$ and $A_{3}$ take values in $L_{2}(-1,0)$.

The system (11) may be represented in a Hilbert space by the equation

$$
\dot{x}(t)=\mathcal{A} x(t)+\mathcal{B} u(t),
$$

where $\mathcal{B} u=(B u, 0)$ and $\mathcal{A}$ is the infinitesimal generator of a $C_{0}$-semigroup $\mathrm{e}^{\mathcal{A} t}$ given in the product space

$$
M_{2}\left(-1,0 ; \mathbb{R}^{n}\right) \stackrel{\text { def }}{=} \mathbb{R}^{n} \times L_{2}\left(-1,0 ; \mathbb{R}^{n}\right),
$$

${ }^{*}$ Corresponding author briefly denoted by $M_{2}$ and defined by

$$
\mathcal{A} x(t)=\left(\begin{array}{c}
L z_{t}(\cdot) \\
\frac{\mathrm{d} z_{t}(\theta)}{\mathrm{d} \theta}
\end{array}\right), \quad x(t)=\left(\begin{array}{c}
v(t) \\
z_{t}(\cdot)
\end{array}\right),
$$

with the domain $D(\mathcal{A})$ given by

$$
\begin{aligned}
D(\mathcal{A})= & \left\{(v, \varphi): \varphi(\cdot) \in H^{1},\right. \\
& \left.v=\varphi(0)-A_{-1} \varphi(-1)\right\} .
\end{aligned}
$$

Our purpose is to analyze exact null controllability of delay systems of neutral type (11), to show the relation with complete stabilizability (exponential stabilizability with an arbitrary decay rate) of the system and, by duality, to give conditions for exact final observability of such a system with an output $y(t)=C z(t)$ or $y(t)=C z(t-1)$, where $y(t)$ takes values in $\mathbb{R}^{p}$.

The problem of exact controllability for systems of neutral type has been widely investigated. References and important results for the system (1) can be found in 
the work of Rabah and Sklyar (2007). A simplification and improvement of some details of the proofs are given by Rabah et al. (2016). Duality with exact (continuous) observability is analyzed by Rabah and Sklyar (2016). For the stabilizability problem, after the first important works (Pandolfi, 1976; O'Connor and Tarn, 1983), there have been many results on the stabilizability of delay systems (see, for example, the works of Richard (2003) or Michiels and Niculescu (2007) and the references therein), but neutral type systems have been less frequently investigated (Pritchard and Salamon, 1987; Salamon, 1984). In the work of Hale and Verduyn Lunel (2002) the main scheme of stabilizing neutral type systems and the robustness (with respect to the delays) of the stabilizing feedback were analyzed. The problem of asymptotic nonexponential stabilizability, which appears only for neutral type systems, was treated by Rabah et al. (2008; 2012); this problem occurs for some systems governed by partial differential equations (see, e.g., Sklyar and Szkibiel, 2013).

This paper is organized as follows. In Section 2 we give results on the relation between exact null controllability and complete stabilizability for abstract systems in Hilbert spaces. In Section 3, we give necessary conditions of exact null controllability and we characterize complete stabilizability for neutral type systems. Then we formulate a conjecture on the equivalence between exact null controllability and complete stabilizability for neutral type systems. Section 4 is concerned with the dual notion of observability: final continuous observability.

\section{Preliminary results}

In this section we consider the abstract system

$$
\dot{x}=\mathcal{A} x+\mathcal{B} u,
$$

where the linear operator $\mathcal{A}$, with domain $D(\mathcal{A})$, is the infinitesimal generator of a $C_{0}$-semigroup $\mathrm{e}^{\mathcal{A} t}$ in the Hilbert space $X$ and $\mathcal{B}$ is a linear operator, which may be unbounded but admissible (see, e.g., Tucsnak and Weiss, 2009), from the Hilbert space $U$ to $X$.

2.1. Bounded input and feedback. Let us first suppose that the operator $\mathcal{B}$ is bounded. The solution of the system (2) with the initial condition $x_{0}$ and the control $u(t) \in L_{2}^{\text {loc }}\left(\mathbb{R}^{+} ; U\right)$ is given by

$$
x(t)=\mathrm{e}^{\mathcal{A} t} x_{0}+\int_{0}^{t} \mathrm{e}^{\mathcal{A}(t-\tau)} B u(\tau) \mathrm{d} \tau .
$$

The following notions are well known (see, e.g., Curtain and Zwart, 1995)

Definition 1. The system (2) is said to be exactly controllable at time $T$ if for all $x_{0}, x_{1} \in X$ there is a control $u(t) \in L_{2}(0, T ; U)$ such that the corresponding solution of the system satisfies $x(T)=x_{1}$. The system is said to be exactly null controllable if in the preceding definition $x_{1}=0$.

There are several results on exact (null) controllability. For example, it is well known that if $\mathcal{B}$ is compact, particularly if $U$ is finite dimensional, then there is no exact controllability (first proved by Kuperman and Repin (1971); see also the work of Curtain and Zwart (1995) and the references therein). Another condition of exact controllability, in the case of the bounded operator $\mathcal{B}$, is that for all $t \geq 0$ the operator $\mathrm{e}^{\mathcal{A} t}$ is onto (surjective) (Louis and Wexler, 1983).

In what follows, we need the following criteria of exact (null) controllability (Curtain and Zwart, 1995).

Theorem 1. The system (2) is exactly null controllable at time $T$ if and only if

$$
\exists \delta>0: \forall x \in X, \int_{0}^{T}\left\|B^{*} \mathrm{e}^{\mathcal{A}^{*} t} x\right\|^{2} \mathrm{~d} t \geq \delta^{2}\left\|\mathrm{e}^{\mathcal{A}^{*} T} x\right\|^{2} .
$$

For the condition of exact controllability, the operator $\mathrm{e}^{\mathcal{A}^{*} T}$ must be replaced by the identity I on the right-hand side of the inequality.

The characterization of exact null controllability is due to a result on range inclusion in Hilbert spaces (Douglas, 1966).

We also need some notions of stabilizability.

Definition 2. The system (2) is said to be exponentially stabilizable if there is a linear bounded feedback operator $\mathcal{F}$ such that the semigroup $\mathrm{e}^{(\mathcal{A}+\mathcal{B} \mathcal{F}) t}$ is exponentially stable: there is a $\omega>0$ such that

$$
\left\|\mathrm{e}^{(\mathcal{A}+\mathcal{B} \mathcal{F}) t}\right\| \leq M_{\omega} \mathrm{e}^{-\omega t}, \quad M_{\omega} \geq 1 .
$$

The system is said to be completely stabilizable (or stabilizable with an arbitrary decay rate) if for all $\omega>0$ there is a linear bounded feedback $\mathcal{F}_{\omega}$ such that (3) holds.

The relation between exact controllability and stabilizability is as follows: exact null controllability implies exponential stabilizability. If $\mathrm{e}^{\mathcal{A} t}$ is a group, complete stabilizability implies exact controllability as shown by Zabczyk (1992, Thm. 3.4, p. 229). Note that the original proof was obtained by Zabczyk (1976), who extended the result of Megan (1975). The same result was proved by Rabah and Karrakchou (1997) or Zeng et al. (2013) for the case of a semigroup $\mathrm{e}^{\mathcal{A} t}$ provided that the operators $\mathrm{e}^{\mathcal{A} t}$ are surjective for all $t \geq 0$.

We have been tempted to extend this latter result to exact null controllability, possibly under some additional conditions. However, the situation is not that simple. We have the following implication, but its converse is not true. 
Theorem 2. If the system (2) is exactly null controllable, then it is completely stabilizable by a bounded feedback $\mathcal{F}$.

Proof. Suppose that the system is exactly null controllable at time $T$. Then

$$
\forall x_{0} \in X, \exists u(\cdot) \in L_{2}(0, T ; U): \quad x\left(T, x_{0}, u(\cdot)\right)=0,
$$

where $x(t)=x\left(t, x_{0}, u(\cdot)\right)$ is the solution with the initial condition $x_{0}$ and the control $u(t)$ :

$$
x\left(t, x_{0}, u(\cdot)\right)=\mathrm{e}^{\mathcal{A} t} x_{0}+\int_{0}^{t} \mathrm{e}^{\mathcal{A}(t-\tau)} \mathcal{B} u(\tau) \mathrm{d} \tau .
$$

Then for every $x_{0} \in X$, there exists $u(\cdot) \in L_{2}(0, \infty ; U)$ such that

$$
\int_{0}^{+\infty}\left(\|x(t)\|^{2}+\|u(t)\|^{2}\right) \mathrm{d} t<\infty
$$

This means that the system is exponentially stabilizable (Zabczyk, 1992, Thm. 3.3, p. 227):

$$
\begin{array}{r}
\exists F_{\omega_{0}} \in \mathcal{L}(U, X):\left\|\mathrm{e}^{\left(\mathcal{A}+\mathcal{B} \mathcal{F}_{\omega_{0}}\right) t}\right\| \leq M_{\omega_{0}} \mathrm{e}^{-\omega_{0} t}, \\
\omega_{0}>0 .
\end{array}
$$

On the other hand, the exact null controllability of the system (2) is equivalent to that of the system

$$
\dot{x}=(\mathcal{A}+\omega I) x+\mathcal{B} u, \quad \omega>0 .
$$

This means that for all $\omega>0$, for some $\mu_{\omega}>0$, there is $\mathcal{F}_{\omega} \in \mathcal{L}(U, X)$ such that

$$
\left\|\mathrm{e}^{\left(\mathcal{A}+\mathcal{B} \mathcal{F}_{\omega}\right) t}\right\| \leq M_{\mu_{\omega}} \mathrm{e}^{-\left(\mu_{\omega}+\omega\right) t} \leq M_{\omega} \mathrm{e}^{-\omega t} .
$$

In order to explain the fact that the converse is not true and that the situation is more complicated, we give examples of two systems without control, where the semigroups are exponentially stable with an arbitrary decay rate, but where the states may or may not reach the null state in finite time. These examples can be found in the work of Rhandi (2002) in the spaces of continuous functions.

Example 1. In the space $L_{2}(0,+\infty)$, consider the semigroup

$$
S(t) f(x)=\mathrm{e}^{-\frac{t^{2}}{2}-x t} f(x+t), \quad t \geq 0, \quad x \geq 0 .
$$

It is easy to see that for this semigroup, for all $\omega>0$, there is a constant $M_{\omega} \geq 1$ such that $\|S(t)\| \leq M_{\omega} \mathrm{e}^{-\omega t}$. We have also $\sigma(S(t))=\{0\}$, and then the spectrum of the infinitesimal generator is empty. On the other hand, there are initial conditions $f$ such that $S(t) f \neq 0$ for any $t \geq 0$.
Example 2. In the space $L_{2}(0,1)$, consider the semigroup

$$
S(t) f(x)= \begin{cases}f(x+t), & 0 \leq t+x \leq 1, \\ 0, & t+x>1\end{cases}
$$

It is not difficult to see that for this semigroup, for all $\omega>0$, there is a constant $M_{\omega} \geq 1$ such that $\|S(t)\| \leq$ $M_{\omega} \mathrm{e}^{-\omega t}$. We also have $\sigma(S(t))=\{0\}$, and then the spectrum of the infinitesimal generator is empty. But, for any initial function $f \in L_{2}(0,1)$, we have $S(t) f(x)=0$ for $t>2$. This means that $S(t)=0$ for all $t>2$. Then, for any control operator $\mathcal{B}$, the corresponding system is exactly null controllable at time $T>2$ with the trivial control $u=0$.

2.2. Unbounded input and feedback operators. For some control systems, the input operator $\mathcal{B}$ may not be bounded and it is very restrictive to assume that the feedback operator $\mathcal{F}$ is bounded. For a general theory on systems with unbounded control and observation, we refer to the paper by Salamon (1987). For the subclass of interest, which includes linear neutral type systems, we refer to Pritchard and Salamon (1987), Curtain et al. (1997) and Guo et al. (2003)).

As our final goal is to analyze exact null controllability and complete stabilizability for neutral type systems, we will now consider a wider context of systems with unbounded input and output operators. However, the situation is much more complicated, even if some extension may be considered.

Let $X_{1}$ be $D(\mathcal{A})$ endowed with the graph norm denoted by $\|x\|_{1}$ and $X_{-1}$ be the completion of the space $X$ with respect to the resolvent norm

$$
\|x\|_{-1}=\left\|(\lambda I-\mathcal{A})^{-1} x\right\|_{X}, \quad \lambda \in \rho(A) .
$$

We have the following relation:

$$
X_{1} \subset X \subset X_{-1},
$$

with continuous dense injections.

Definition 3. Let $\mathcal{B}$ be a linear operator, bounded from the Hilbert space $U$ to $X$. We say that $\mathcal{B}$ is an admissible input operator for the semigroup $\mathrm{e}^{\mathcal{A} t}$ if there exists $t_{1}$ such that

$$
\int_{0}^{t_{1}} \mathrm{e}^{\mathcal{A}\left(t_{1}-\tau\right)} \mathcal{B} u(\tau) \mathrm{d} \tau \in X_{1},
$$

and for some $\beta>0$

$$
\left\|\int_{0}^{t_{1}} \mathrm{e}^{\mathcal{A}\left(t_{1}-\tau\right)} \mathcal{B} u(\tau) \mathrm{d} \tau\right\|_{X_{1}} \leq \beta\|u\|_{L_{2}\left(0, t_{1}\right)}
$$


Definition 4. Assume that operator $\mathcal{F}$ is a linear operator, bounded from $X_{1}$ to the Hilbert space $Y$. We say that it is an admissible output operator for the semigroup $\mathrm{e}^{\mathcal{A} t}$ if there exists $t_{1}>0$ such that for some $\alpha>0$

$$
\left\|\mathcal{F} \mathrm{e}^{\mathcal{A}\left(t_{1}-\tau\right)} x\right\|_{L_{2}\left(0, t_{1}\right)} \leq \alpha\|x\|_{X}, \quad x \in X_{1} .
$$

Admissibility for some $t_{1}$ implies admissibility for all $t>0$ (see, e.g., Curtain et al., 1997). From the general result on the perturbation of semigroup from the Pritchard-Salamon class, we can deduce the following Cauchy formula for the perturbed semigroup $\mathrm{e}^{(\mathcal{A}+\mathcal{B} \mathcal{F}) t}$, for admissible input and output operators $\mathcal{B}$ and $\mathcal{F}$ :

$$
\mathrm{e}^{(\mathcal{A}+\mathcal{B} \mathcal{F}) t} x=\mathrm{e}^{\mathcal{A} t} x+\int_{0}^{t} \mathrm{e}^{\mathcal{A}(t-\tau)} \mathcal{B} \mathcal{F} \mathrm{e}^{(\mathcal{A}+\mathcal{B} \mathcal{F}) \tau} x \mathrm{~d} \tau
$$

for all $x \in X_{1}$. Moreover, $\mathrm{e}^{(\mathcal{A}+\mathcal{B} \mathcal{F}) t}$ extends to a $C_{0}$-semigroup on $X$.

This means that Definition 2 may be reformulated for an admissible input operator and an admissible output feedback.

Theorem 3. If the system (2) with an admissible operator $\mathcal{B}$ is completely stabilizable by an admissible $\mathcal{A}$-bounded feedback, then it is completely stabilizable by a bounded linear feedback $\mathcal{F}$.

Proof. In the work of Curtain et al. (1997, Thm. 5.5) (see also Guo et al., 2003), in a more general situation, it is shown that the system (2) with an admissible operator $\mathcal{B}$ is exponentially stabilizable by an admissible feedback (in $X_{1}$ and $X$ ) if and only if it is exponentially stabilizable by a bounded feedback. Thus, we can suppose, with no loss of generality, that in (4) the operator $\mathcal{F}$ is bounded: $\mathcal{F} \in \mathcal{L}(X, U)$. This means that complete stabilizability by admissible feedbacks holds if and only if there is a complete stabilizability by bounded feedbacks.

From this and Theorem 2] we can expect to extend the result of Theorem 2 to the case of unbounded control and feedback. But Theorem 2 is based on the result of Zabczyk (1992, Thm. 3.3, p. 227), which needs another (Zabczyk, 1992, Thm. 4.3, p. 240), based on the assumption of the exact null controllability given by Definition 1. For the case of unbounded control and feedback, we refer to Pritchard and Salamon (1987, Thm. 3.3 , p. 132). The condition $\mathrm{H} 4$ used in this theorem is guaranteed by exact null controllability in $X_{-1}$ (each initial state from $X_{-1}$ may be moved to zero by an $L_{2}$ control).

Corollary 1. If the system (2) with an admissible operator $\mathcal{B}$ is exactly null controllable in $X_{-1}$, then it is completely stabilizable by an admissible feedback and then by a bounded feedback $\mathcal{F}$.
2.3. Technical lemma. In the next section we need the following lemma.

Lemma 1. Let $A$ be an $(n \times n)$-matrix and $B$ an $(n \times m)$ matrix. The following statements are equivalent

(i) For all $\lambda \in \mathbb{C}, \lambda \neq 0, \operatorname{rank}(\lambda I-A \quad B)=n$.

(ii) The following equality holds:

$$
\begin{aligned}
\operatorname{rank} & \left(\begin{array}{lllll}
B & A B & \cdots & A^{n-1} B
\end{array}\right) \\
& =\operatorname{rank}\left(\begin{array}{lllll}
B & A B & \cdots & A^{n-1} B & A^{n}
\end{array}\right),
\end{aligned}
$$

and this is equivalent to the inclusion

$$
\operatorname{Im} A^{n} \subset \operatorname{Im}\left(\begin{array}{llll}
B & A B & \cdots & A^{n-1} B
\end{array}\right) .
$$

Proof. The conditions 1 and 2 may be formulated as follows:

(i) If there is $x \neq 0$ such that $A^{*} x=\lambda x$ and $B^{*} x=0$, then $\lambda=0$.

(ii) If $x \neq 0$ is such that $B^{*} A^{* i} x=0, i \in \mathbb{N}$, then $A^{* n} x=0$.

Suppose that (i) holds. Let $\mathcal{N}$ be the subspace

$$
\mathcal{N}=\left\{x: B^{*} A^{* i} x=0, i \in \mathbb{N}\right\} .
$$

It is easy to see that $\mathcal{N}$ is $A^{*}$-invariant and contained in Ker $B^{*}$. The spectrum of the restriction of $A^{*}$ to $\mathcal{N}$ is $\{0\}$ by the condition (i). This means that $A^{*}$ is nilpotent in $\mathcal{N}$. As the dimension of $\mathcal{N}$ is $k \leq n$, we obtain $A^{* n} x=0$ for all $x \in \mathcal{N}$. This gives (ii).

Let us show the equivalence of these conditions. Suppose now that (ii) holds. Let $x \neq 0$ be such that $A^{*} x=\lambda x$ and $B^{*} x=0$. This implies that $B^{*} A^{* i} x=$ $\lambda^{i} B^{*} x=0$, for all $i \in \mathbb{N}$. From (ii), we obtain that

$$
0=A^{* n} x=\lambda^{n} x .
$$

As $x \neq 0$, this implies $\lambda=0$ and yields (i).

\section{Neutral type system: Controllability and stabilizability}

In this section we analyze exact null controllability and complete stabilizability (exponential stabilizability with an arbitrary decay rate) of a delay system of neutral type (1) and investigate the relation between the two notions. By duality, we give conditions for exact final observability of such system with outputs

$$
y(t)=C z(t) \quad \text { or } \quad y(t)=C z(t-1),
$$

where $y(t)$ takes values in $\mathbb{R}^{p}$.

The relation between exact controllability and exponential stabilizability for linear neutral type systems may 
be found in several papers (see, e.g., the works of Salamon (1984), Ito and Tarn (1985), O'Connor and Tarn (1983) or Dusser and Rabah (2001) and the references therein).

For the analysis of stabilizability, we need the structure of the spectrum of the state operator $\mathcal{A}$ of the system (1) and the condition of the growth of semigroup $\mathrm{e}^{\mathcal{A} t}$.

Theorem 4. (Rabah et al., 2005) Consider the matrix

$$
\begin{aligned}
\Delta_{\mathcal{A}}(\lambda)= & \lambda I-\lambda e^{-\lambda} A_{-1} \\
& -\int_{-1}^{0}\left[\lambda e^{\lambda s} A_{2}(s)+e^{\lambda s} A_{3}(s)\right] \mathrm{d} s .
\end{aligned}
$$

The spectrum of $\mathcal{A}$, denoted by $\sigma(\mathcal{A})$, consists only of the eigenvalues which are the roots of the equation $\operatorname{det} \Delta_{\mathcal{A}}(\lambda)=0$. The corresponding eigenvectors of $\mathcal{A}$ are of the form

$$
\left(\begin{array}{c}
v-e^{-\lambda} A_{-1} v \\
e^{\lambda \theta} v
\end{array}\right), \quad v \in \operatorname{Ker} \Delta_{\mathcal{A}}(\lambda) .
$$

The spectrum of $\mathcal{A}$ contains a nonempty set of points of the form

$$
\{\ln |\mu|+\mathrm{i}(\arg \mu+2 \pi k)+O(1 / k), k \in \mathbb{Z}\},
$$

where $\mu$ is a non-zero eigenvalue of the matrix $A_{-1}$.

The spectrum is countable and the semigroup $\mathrm{e}^{\mathcal{A} t}$ satisfies the spectrum growth assumption (see, e.g., Hale and Verduyn Lunel, 1993)

forall $\omega>\omega_{0}=\sup \operatorname{Re} \sigma(\mathcal{A}), \quad \exists M_{\omega}:\left\|\mathrm{e}^{\mathcal{A} t}\right\| \leq M_{\omega} \mathrm{e}^{\omega t}$.

Definition 5. System (1) is exactly null controllable if for some $T>0$ and for all $x_{0} \in M_{2}$ there is a control $u(\cdot) \in L_{2}\left(0, T ; \mathbb{R}^{m}\right)$ such that

$$
\mathrm{e}^{\mathcal{A} t} x_{0}+\int_{0}^{T} \mathrm{e}^{\mathcal{A}(T-\tau)} \mathcal{B} u(\tau) \mathrm{d} \tau=0 .
$$

This notion corresponds to the concept of complete controllability given first by N.N. Krasovskiǐ for retarded systems.

Let $\mathcal{R}_{T}$ be the linear operator defined by

$$
\begin{aligned}
& \mathcal{R}_{T} u(\cdot)=\int_{0}^{T} \mathrm{e}^{\mathcal{A}(T-\tau)} \mathcal{B} u(\tau) \mathrm{d} \tau, \\
& u(\cdot) \in L_{2}\left(0, T ; \mathbb{R}^{m}\right) .
\end{aligned}
$$

The operator $\mathcal{R}_{T}$ is bounded from $L_{2}\left(0, T ; \mathbb{R}^{m}\right)$ to $X$. Moreover, it takes values in $D(\mathcal{A})$ and is bounded from $L_{2}\left(0, T ; \mathbb{R}^{m}\right)$ to $X_{1}$ (see the works of Ito and Tarn (1985, Corollary 2.7) and Rabah and Sklyar (2007) for our system).
The exact null controllability may be formulated by the inclusion

$$
\operatorname{Im} \mathrm{e}^{\mathcal{A} T} \subset \operatorname{Im} \mathcal{R}_{T},
$$

where $\operatorname{Im} \mathrm{e}^{\mathcal{A} T}$ and $\operatorname{Im} \mathcal{R}_{T}$ are images of the operators $\mathrm{e}^{\mathcal{A} T}$ and $\mathcal{R}_{T}$. From the well-known characterization of range inclusion in Hilbert spaces (Douglas, 1966), we can obtain the following proposition, which is an extension of Theorem 1

Proposition 1. The system (11) is exactly null controllable for some $T>0$ if and only if there is a constant $\delta>0$ such that

$$
\int_{0}^{T}\left\|\mathcal{B}^{*} \mathrm{e}^{\mathcal{A}^{*}(T-\tau)} x\right\|_{\mathbb{R}^{m}}^{2} \mathrm{~d} \tau \geq \delta^{2}\left\|\mathrm{e}^{\mathcal{A}^{*} T} x\right\|_{M_{2}}^{2},
$$

for all $x \in M_{2}$.

We can now give the main result of this section.

Theorem 5. If the system (1) is exactly null controllable, then the following two conditions hold:

(i) $\operatorname{rank}\left(\Delta_{\mathcal{A}}(\lambda) \quad B\right)=$ n for all $\lambda \in \mathbb{C}$,

(ii) $\operatorname{rank}\left(\mu I-A_{-1} \quad B\right)=n$ for all $\mu \in \mathbb{C}, \mu \neq 0$.

Proof. Suppose that the system (1) is exactly null controllable. The necessity of the condition (i) is trivial. Let us show that the condition (ii) is satisfied. We follow a method used by Metel'skiı̌ and Minyuk (2006) (see also Khartovskiĭ and Pavlovskaya, 2013). Then, for some $T$, for all initial conditions, in particular for all $\varphi \in$ $H^{1}\left(-1,0 ; \mathbb{R}^{n}\right)$, there is a control $u(\cdot) \in L_{2}(0, T ; \mathbb{R})$, $u(t)=0$ for $t>T$, such that $z(t)=0, t>T$. We may suppose that $T>n$.

The function $z(t)$ is absolutely continuous and hence almost everywhere differentiable. Then we have

$$
\dot{z}(t)=A_{-1} \dot{z}(t-1)+L z_{t}+B u(t) .
$$

Replacing $\dot{z}(t-1)$ in this equation, we obtain

$\dot{z}(t)=A_{-1}\left(A_{-1} \dot{z}(t-2)+L z_{t-1}+B u(t-1)\right)+B u(t)$.

With no loss of generality, one can suppose that the time $t$ is such that the function $u$ is well defined at these points. Repeating this procedure, we obtain

$$
\begin{aligned}
\dot{z}(t)= & A_{-1}^{N} \dot{z}(t-N) \\
& +\sum_{k=0}^{N-1} A_{-1}^{k}\left(L z_{t-k}+B u(t-k)\right) .
\end{aligned}
$$

Setting $t=N \geq T$ and using the continuity of $z(t)$, we obtain

$$
\begin{aligned}
0 & =A_{-1}^{N}(\dot{z}(+0)-\dot{z}(-0)) \\
& +\sum_{k=0}^{N-1} A_{-1}^{k}(B u(N-k+0)-B u(N-k-0)) .
\end{aligned}
$$


As $z(t)$ for $t>0$ is the solution of Eqn. (1), we have

$$
\dot{z}(+0)=A_{-1} \dot{z}(-1)+L z_{+0}(\cdot)+B u(+0) .
$$

Then, replacing this expression in (5) and setting the initial condition $z_{0}(\theta)=\varphi(\theta)$, we obtain

$$
\begin{aligned}
& A_{-1}^{N}\left(A_{-1} \dot{\varphi}(-1)+L \varphi(\theta)-\dot{\varphi}(-0)\right)+A_{-1}^{N} B u(+0) \\
& +\sum_{k=0}^{N-1} A_{-1}^{k}(B u(N-k+0)-B u(N-k-0))=0 .
\end{aligned}
$$

As $\dot{\varphi}(-0) \in \mathbb{R}^{n}$ may be chosen arbitrarily, we obtain

$$
\operatorname{Im} A_{-1}^{N} \subset \operatorname{Im}\left(\begin{array}{llll}
B & A_{-1} B & \cdots & A_{-1}^{N-1} B
\end{array}\right) .
$$

This may be written as

$$
\begin{aligned}
\operatorname{rank} & \left(\begin{array}{lllll}
B & A_{-1} B & \cdots & A_{-1}^{N-1} B
\end{array}\right) \\
& =\operatorname{rank}\left(\begin{array}{llllll}
B & A_{-1} B & \cdots & A_{-1}^{N-1} B & A_{-1}^{N}
\end{array}\right) .
\end{aligned}
$$

By the Cayley-Hamilton theorem, this gives

$$
\begin{aligned}
\operatorname{rank} & \left(\begin{array}{lllll}
B & A_{-1} B & \cdots & A_{-1}^{n-1} B
\end{array}\right) \\
& =\operatorname{rank}\left(\begin{array}{lllll}
B & A_{-1} B & \cdots & A_{-1}^{n-1} B & A_{-1}^{n}
\end{array}\right) .
\end{aligned}
$$

Now, using Lemma 1 we obtain the condition (ii).

The necessary conditions of exact null controllability characterize in fact the property of complete stabilizability.

Theorem 6. The system (1) is completely stabilizable by a feedback law of the form

$$
u(t)=F_{-1} \dot{z}(t-1)+F z_{t}(\cdot)
$$

where

$$
F z_{t}(\cdot)=\int_{-1}^{0}\left[F_{2}(\theta) \dot{z}(t+\theta)+F_{3}(\theta) z(t+\theta)\right] \mathrm{d} \theta
$$

if and only if

(i) $\operatorname{rank}\left(\Delta_{\mathcal{A}}(\lambda) \quad B\right)=n$ for all $\lambda \in \mathbb{C}$,

(ii) $\operatorname{rank}\left(\mu I-A_{-1} \quad B\right)=n$ for all $\mu \in \mathbb{C}, \mu \neq 0$.

Proof. We give a short and direct proof of the necessity even though it can be obtained from Corollary 5.1.3 of Salamon (1984).

If the condition (i) is not satisfied then there is an eigenvalue $\lambda_{0}$ of the operator $\mathcal{A}$ which cannot be modified by the control operator $\mathcal{B}$. This implies the lack of complete stabilizability.

If the condition (ii) is not satisfied, then there is a nonzero eigenvalue $\mu_{0}$ of the matrix $A_{-1}$ which cannot be modified. Then the spectral set

$\left\{\ln \left|\mu_{0}\right|+\mathrm{i}\left(\arg \mu_{0}+2 \pi k\right)+O(1 / k), k \in \mathbb{Z}\right\} \subset \sigma(\mathcal{A})$, which belongs to a vertical strip, cannot be modified either. This means that complete stabilizability is not possible.

Let us show now that the two conditions are sufficient for complete stabilizability feedback laws of the form (6).

Suppose that the condition (ii) is satisfied. Let us fix an arbitrary $\omega>0$. As all the non-zero poles of the matrix $A_{-1}$ are controllable by the condition (ii), a matrix $F_{-1}$ can be found such that the spectrum $\sigma\left(A_{-1}+B F_{-1}\right)$ satisfies

$$
\forall \mu \in \sigma\left(A_{-1}+B F_{-1}\right), \quad \mu \neq 0, \quad \ln |\mu|<-\omega .
$$

Consider now the neutral type system

$$
\dot{z}(t)=\left(A_{-1}+B F_{-1}\right) \dot{z}(t-1)+L z_{t}+B u .
$$

Let $\mathcal{A}_{1}$ be the generator of the system (7). From the structure of the spectrum of neutral type systems like (1), we have only a finite number of eigenvalues $\lambda \in \sigma\left(\mathcal{A}_{1}\right)$ such that $\operatorname{Re} \lambda \geq-\omega$. Now, using the condition (i), a feedback $u(t)=F_{1} z_{t}(\cdot)$, where

$$
F_{1} z_{t}(\cdot)=\int_{-1}^{0}\left[F_{2}(\theta) \dot{z}(t+\theta)+F_{3}(\theta) z(t+\theta)\right] \mathrm{d} \theta,
$$

can be found (see, e.g., Pandolfi, 1976; Pritchard and Salamon, 1987; Rabah et al., 2008; 2012) such that all the eigenvalues $\lambda$ of the system

$$
\dot{z}(t)=\left(A_{-1}+B F_{-1}\right) \dot{z}(t-1)+\left(L+B F_{1}\right) z_{t}
$$

satisfy $\operatorname{Re} \lambda<-\omega$. If we denote by $\mathcal{F}$ the global feedback

$$
\left.u(t)=F_{-1} \dot{z}(t-1)+F_{1} z_{t}(\cdot)\right)
$$

then we obtain

$$
\left\|\mathrm{e}^{(\mathcal{A}+\mathcal{B} \mathcal{F}) t}\right\| \leq M \mathrm{e}^{-\omega t}, \quad M \geq 1 .
$$

Since $\omega$ has been arbitrarily taken, this means that the system is completely stabilizable by a feedback of the form (6).

A similar result was obtained for modal controllability (the assignment of a characteristic quasi-polynomial) for a neutral system with multiple discrete delays by Metel'skiǔ and Khartovskii (2016).

In view of Corollary 1 as well as Theorems 5 and 6 . one can formulate the following natural conjecture.

Conjecture 1. The system (1) is exactly null controllable if the following two conditions hold:

(i) $\operatorname{rank}\left(\Delta_{\mathcal{A}}(\lambda) \quad B\right)=$ n for all $\lambda \in \mathbb{C}$,

(ii) $\operatorname{rank}\left(\mu I-A_{-1} \quad B\right)=n$ for all $\mu \in \mathbb{C}, \mu \neq 0$. 
This means that exact null controllability is equivalent to complete stabilizability for neutral type systems.

It is well known that Conjecture 1 is satisfied for some class of neutral type systems with discrete delays (Metel'skiı̌ and Minyuk, 2006; Khartovskiı̌ and Pavlovskaya, 2013) and in the case of retarded systems (Olbrot and Pandolfi, 1988). It seems that one can use the conditions of complete stabilizability to show the result of Conjecture 1. But at this moment, we not have a satisfactory formal proof.

\section{Final exact observability}

The dual notion of exact null controllability in a Hilbert space is the notion of final continuous observability. Sometimes the term continuous is replaced (by analogy) by the term exact. In the work of Rabah and Sklyar (2016), the duality between exact controllability and exact observability was analyzed. In the present section we give the result for null exact controllability and the corresponding notion of observability.

We consider the finite dimensional observation

$$
y(t)=\mathcal{C} x(t),
$$

where $\mathcal{C}$ is a linear operator and $y(t) \in \mathbb{R}^{p}$ is a finite dimensional output. There are several ways to design the output operator $\mathcal{C}$ (Salamon, 1983; 1984; Metel'skii and Minyuk, 2006). One of our goals in this paper is to investigate how to design a minimal output operator like

$$
\mathcal{C} x(t)=C z(t) \quad \text { or } \quad \mathcal{C} x(t)=C z(t-1),
$$

where $C$ is a $p \times n$ matrix. More general outputs, for example, with several and/or distributed delays, are not considered here. We want to use some results on exact controllability in order to analyze, by duality, the exact observability property in the infinite dimensional setting like, for example, in the work of Tucsnak and Weiss (2009).

The operator $\mathcal{C}$ defined in (9) is linear but not bounded in $M_{2}$. However, in both the cases it is admissible in the following sense:

$$
\int_{0}^{T}\left\|\mathcal{C} \mathrm{e}^{\mathcal{A} t} x_{0}\right\|_{\mathbb{R}^{p}}^{2} \mathrm{~d} t \leq \kappa^{2}\left\|x_{0}\right\|_{M_{2}}^{2}, \quad \forall x_{0} \in D(\mathcal{A}),
$$

because $\mathrm{e}^{\mathcal{A} t} x_{0} \in D(\mathcal{A}), t \geq 0$ (see, e.g., Pazy, 1983).

Definition 6. Let $\mathcal{K}$ be the output operator

$$
\mathcal{K}: M_{2} \longrightarrow L_{2}\left(0, T ; \mathbb{R}^{p}\right), \quad x_{0} \longmapsto \mathcal{K} x_{0}=\mathcal{C} \mathrm{e}^{\mathcal{A} t} x_{0} .
$$

The system (1) is said to be exactly finally observable or continuously finally observable (Salamon, 1984) if

$$
\left\|\mathcal{K} x_{0}\right\|_{L_{2}}^{2}=\int_{0}^{T}\left\|\mathcal{C} \mathrm{e}^{\mathcal{A} t} x_{0}\right\|_{\mathbb{R}^{p}}^{2} \mathrm{~d} t \geq \gamma^{2}\left\|\mathrm{e}^{\mathcal{A} T} x_{0}\right\|_{M_{2}}^{2}
$$

for some constant $\gamma>0$ and for all $x_{0} \in D(\mathcal{A})$. We say that the system is exactly (or continuously) observable if in (6) in the second term of the inequality, $\mathrm{e}^{\mathcal{A} T} x_{0}$, is replaced by $x_{0}$.

Exact observability means that we can continuously determine the initial state $z_{0}(\cdot)$ from the observation on $[0, T]$. Final exact observability signifies that we can continuously determine the final state $z_{T}(\cdot)$.

Exact (final) observability depends essentially on the space topology. We can expect that a given neutral type system is not exactly observable if we consider $x_{0} \in$ $D(\mathcal{A})$ with the graph norm and no longer in the topology of $M_{2}$. In fact, we obtain the final observability in the initial norm, but we need some delay in the observation in the general case.

In order to use the duality between observability and controllability, we need the expression of the adjoint operator $\mathcal{K}^{*}$ in the duality with respect to the pivot space $M_{2}$ in the embedding

$$
X_{1} \subset X=M_{2} \subset X_{-1} \text {, }
$$

where $X_{1}=D(\mathcal{A})$ with the graph norm denoted by $\|x\|_{1}$ and $X_{-1}$ being the completion of the space $M_{2}$ with respect to the resolvent norm $\|x\|_{-1}=$ $\left\|(\lambda I-\mathcal{A})^{-1} x\right\|_{M_{2}}$. The duality relation is

$$
\left\langle\mathcal{K} x_{0}, u(\cdot)\right\rangle_{L_{2}\left(0, T ; \mathbb{R}^{p}\right)}=\left\langle x_{0}, \mathcal{K}^{*} u(\cdot)\right\rangle_{X_{1}, X_{-1}^{\mathrm{d}}},
$$

where $X_{-1}^{\mathrm{d}}$ is constructed as $X_{-1}$ with $\mathcal{A}^{*}$ instead of $\mathcal{A}$ (see Tucsnak and Weiss, 2009) : $X_{-1}^{\mathrm{d}}$ is the completion of the space $M_{2}$ with the resolvent norm corresponding to the operator $\mathcal{A}^{*}$.

Exact null controllability is dual with exact final observability in the corresponding spaces and with the corresponding topologies. It is expected that the operator $\mathcal{K}^{*}$ corresponds to a control operator for some adjoint system. However, the situation is not so simple, as was pointed out in the paper by Rabah and Sklyar (2016), from which we take our main considerations on duality.

Proposition 2. (Rabah et al., 2008; Rabah and Sklyar, 2016) The adjoint operator $\mathcal{A}^{*}$ is given by

$$
\mathcal{A}^{*}\left(\begin{array}{c}
w \\
\psi(\cdot)
\end{array}\right)=\left(\begin{array}{c}
\left(A_{2}^{*}(0) w+\psi(0)\right. \\
-\frac{\mathrm{d}\left[\psi(\theta)+A_{2}^{*}(\theta) w\right]}{\mathrm{d} \theta}+A_{3}^{*}(\theta) w
\end{array}\right),
$$

with the domain $D\left(\mathcal{A}^{*}\right)$ consisting of $(w, \psi(\cdot)) \in M_{2}$ such that

$$
\left\{\begin{array}{l}
\psi(\theta)+A_{2}^{*}(\theta) w \in H^{1}, \\
A_{-1}^{*}\left(A_{2}^{*}(0) w+\psi(0)\right)=\psi(-1)+A_{2}^{*}(-1) w .
\end{array}\right.
$$

Let $x$ be a solution of the abstract equation

$$
\dot{x}=\mathcal{A}^{*} x, \quad x(t)=\left(\begin{array}{c}
w(t) \\
\psi_{t}(\theta)
\end{array}\right) .
$$


Then the function $w(t)$ is the solution of the neutral type equation

$$
\begin{aligned}
\dot{w}(t+1)= & A_{-1}^{*} \dot{w}(t)+\int_{-1}^{0}\left[A_{2}^{*}(\tau) \dot{w}(t+1+\tau)\right. \\
& \left.+A_{3}^{*}(\tau) w(t+1+\tau)\right] \mathrm{d} \tau .
\end{aligned}
$$

This means that the form of the adjoint system is not a simple transposition of the initial one (1). Let us now specify the relation between the solutions of the neutral type equation (12) related to the adjoint system (11) and the transposed neutral type equation

$$
\begin{aligned}
\dot{z}(t)= & A_{-1}^{*} \dot{z}(t-1) \\
& +\int_{-1}^{0}\left[A_{2}^{*}(\tau) \dot{z}(t+\tau), A_{3}^{*}(\tau) z(t+\tau)\right] \mathrm{d} \tau,
\end{aligned}
$$

with initial $z_{0}(\theta)$. Let $\mathcal{A}^{\dagger}$ be the infinitesimal generator of the semigroup corresponding to Eqn. (13).

Set

$$
\left(\begin{array}{c}
w(t) \\
\psi_{t}(\theta)
\end{array}\right)=\mathrm{e}^{\mathcal{A}^{*} t} \xi_{0}=\mathrm{e}^{\mathcal{A}^{*} t}\left(\begin{array}{c}
w(0) \\
\psi_{0}(\theta)
\end{array}\right)
$$

and the conditions

$$
\left(\begin{array}{c}
v(t) \\
z_{t}(\theta)
\end{array}\right)=\left(\begin{array}{c}
w(t+1)-A_{-1}^{*} w(t) \\
w(t+1+\theta)
\end{array}\right)=\mathrm{e}^{\mathcal{A}^{\dagger} t}\left(\begin{array}{c}
v(0) \\
z_{0}(\theta)
\end{array}\right),
$$

where $z_{0}(\theta)=w(\theta+1)$ and $v(0)=z_{0}(0)-A_{-1} z_{0}(-1)$. We can give the explicit relation between the initial conditions $\xi_{0}$ and $x_{0}$ :

$$
\xi_{0}=\left(\begin{array}{c}
w(0) \\
\psi_{0}(\theta)
\end{array}\right), \quad x_{0}=\left(\begin{array}{c}
v(0) \\
z_{0}(\theta)
\end{array}\right)
$$

The formal relation between these vectors is

$$
\xi_{0}=\left(\begin{array}{c}
w(0) \\
\psi_{0}(\theta)
\end{array}\right)=\Phi x_{0}=\Phi\left(\begin{array}{c}
w(1)-A_{-1} w(0) \\
w(\theta+1)
\end{array}\right),
$$

and we have the following result.

Theorem 7. (Rabah and Sklyar, 2016) The operator $\Phi$ representing the relation between the initial conditions $x_{0}$ and $\xi_{0}$ corresponding to the neutral type systems (11)(12) and (13) is linear bounded and bounded invertible from $X_{1}^{\mathrm{d}}$ to $M_{2}$, where $X_{1}^{\mathrm{d}}$ is $D\left(\mathcal{A}^{*}\right)$ with the graph norm.

Let us now consider the reachability operator of the transposed controlled system:

$$
\dot{x}(t)=\mathcal{A}^{\dagger} x(t)+\mathcal{C}^{\dagger} u(t),
$$

where

$$
\mathcal{C}^{\dagger}=\left(\begin{array}{c}
C^{*} \\
0
\end{array}\right)
$$

This operator is given by

$$
\mathcal{R}_{T}^{\dagger} u(\cdot)=\int_{0}^{T} \mathrm{e}^{\mathcal{A}^{\dagger}(T-\tau)} \mathcal{C}^{\dagger} u(\tau) \mathrm{d} \tau
$$

The operator $\mathcal{K}$ may be written using $\mathcal{R}_{T}^{\dagger}$ and the semigroup $\mathrm{e}^{\mathcal{A}^{\dagger}}$ of the system (13) as follows (see Rabah and Sklyar, 2016):

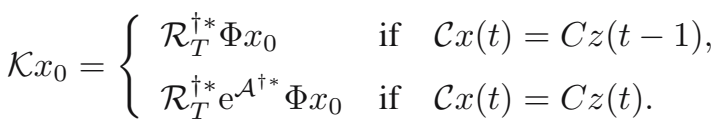

We can now formulate the main result of this section.

Theorem 8. The system (1) with the output $y=C z(t-1)$ is exactly (continuously) finally observable if and only if the system (13) is exactly null controllable. A necessary condition of exact final observability is given by two conditions:

(i) $\operatorname{Ker}\left(\begin{array}{c}\Delta_{\mathcal{A}}(\lambda) \\ C\end{array}\right)=\{0\}$ for all $\lambda \in \mathbb{C}$,

(ii) $\operatorname{Ker}\left(\begin{array}{c}\lambda I-A_{-1} \\ C\end{array}\right)=\{0\}$ for all $\lambda \in \mathbb{C}, \lambda \neq 0$.

Proof. According to the relation (14), we have

$$
\left\|\mathcal{K} x_{0}\right\|_{L_{2}}=\left(\int_{0}^{T}\left\|C^{*} \mathrm{e}^{\mathcal{A}^{\dagger *}(T-\tau)} \Phi x_{0}\right\|^{2} \mathrm{~d} \tau\right)^{\frac{1}{2}} .
$$

As the system (13) is exactly null controllable, we obtain

$$
\left\|\mathcal{K} x_{0}\right\|_{L_{2}} \geq \delta\left\|\mathrm{e}^{\mathcal{A}^{\dagger *} T} \Phi x_{0}\right\|
$$

for all $x_{0} \in D(\mathcal{A})$. It is easy to see from the work of Rabah and Sklyar (2016) that

$$
\mathrm{e}^{\mathcal{A}^{\dagger *} T} \Phi x_{0}=\Phi \mathrm{e}^{\mathcal{A}^{\dagger *}(T-\tau)} x_{0}=\mathrm{e}^{\mathcal{A} T} x_{0}
$$

This gives

$$
\left\|\mathcal{K} x_{0}\right\|_{L_{2}} \geq \delta\left\|\mathrm{e}^{\mathcal{A} T} x_{0}\right\|,
$$

which means that exact final observability holds.

For the case of the output $y=C z(t)$, we cannot say anything if $\operatorname{det}\left(\mathcal{A}_{-1}\right)=0$. If $\mathcal{A}_{-1}$ is not singular, then $\mathrm{e}^{\mathcal{A} t}$ is a group and exact final observability coincides with exact observability (Rabah and Sklyar, 2016).

\section{Examples}

To illustrate our results and hypothesis, we give here three examples. The first one shows that for continuous observability a delay in the output is needed if the semigroup is not a group. The second one is taken from the work of Metel'skil and Minyuk (2006), and it is shown that in fact we have exact controllability (not only exact 
null controllability). The last example illustrates our Conjecture 1 on equivalence between exact controllability and complete stabilizability.

All examples are given in the form of a system with one discrete delay:

$$
\dot{z}(t)=A_{-1} z(t-1)+A_{0} z(t)+A_{1} z(t-1)+B u(t) .
$$

Example 3. We have the system with

$$
\begin{array}{rlrl}
A_{0} & =\left(\begin{array}{ll}
0 & 1 \\
0 & 0
\end{array}\right), & A_{1}=0, \\
A_{-1}=\left(\begin{array}{ll}
0 & 1 \\
0 & 0
\end{array}\right), & B=\left(\begin{array}{l}
0 \\
1
\end{array}\right) .
\end{array}
$$

It is easy to see that, for all $\lambda \in \mathbb{C}$,

$$
\operatorname{rank}\left(\Delta_{\mathcal{A}}(\lambda) \quad B\right)=\left(\begin{array}{ccc}
\lambda & -\lambda \mathrm{e}^{-\lambda}-1 & 0 \\
0 & \lambda & 1
\end{array}\right)=2 .
$$

Moreover, for all $\lambda \in \mathbb{C}$, $\operatorname{rank}\left(\lambda I-A_{-1} \quad B\right)=n$; then the system is exactly controllable (not only to zero). The transposed system

$$
\left\{\begin{array}{l}
\dot{z}_{1}(t)=0 \\
\dot{z}_{2}(t)=\dot{z}_{1}(t-1)+z_{1}(t)
\end{array}\right.
$$

is continuously observable with the output $y=z_{2}(t-1)$ but not with $y(t)=z_{2}(t)$.

Example 4. The following system was given for exact null controllability and continuous final observability by Metel'skiı̌ and Minyuk (2006):

$$
A_{0}=0, \quad A_{1}=\left(\begin{array}{ll}
0 & 1 \\
0 & 0
\end{array}\right), \quad A_{-1}=\left(\begin{array}{cc}
0 & -1 \\
0 & 1
\end{array}\right) .
$$

In fact, for this system, the initial condition is exactly observable by the output

$$
y=C z(t-1), \quad C=\left(\begin{array}{ll}
1 & 0
\end{array}\right),
$$

and the transposed system is exactly controllable because, for all $\lambda \in \mathbb{C}$,

$$
\operatorname{rank}\left(\lambda I-A_{-1}^{*} \quad C^{*}\right)=\operatorname{rank}\left(\begin{array}{ccc}
\lambda & 0 & 1 \\
1 & \lambda-1 & 0
\end{array}\right)=2 .
$$

However, the initial system is not exactly observable by the output $y=C z(t)=z_{1}(t)$, because the initial function $z_{0}(\theta), \theta \in[0,1[$ cannot be determined.

Example 5. We have the system (15) with

$$
\begin{array}{rlrl}
A_{0} & =\left(\begin{array}{ll}
0 & 0 \\
1 & 0
\end{array}\right), & A_{1}=0 \\
A_{-1}=\left(\begin{array}{ll}
1 & 0 \\
0 & 0
\end{array}\right), & B=\left(\begin{array}{l}
1 \\
0
\end{array}\right) .
\end{array}
$$

For all $\lambda \in \mathbb{C}$, we get

$$
\operatorname{rank}\left(\Delta_{\mathcal{A}}(\lambda) \quad B\right)=\left(\begin{array}{ccc}
\lambda-\lambda \mathrm{e}^{-\lambda} & 0 & 1 \\
-1 & \lambda & 0
\end{array}\right)=2,
$$

and, for all complex $\lambda \neq 0$,

$$
\operatorname{rank}\left(\lambda I-A_{-1} \quad B\right)=\left(\begin{array}{ccc}
\lambda-1 & 0 & 1 \\
0 & \lambda & 0
\end{array}\right)=2 .
$$

The system is exactly null controllable by Lemma 1 and the result of Metel'skii and Minyuk (2006). It is completely stabilizable by Theorem 6 Consider now the transposed system

$$
\left\{\begin{array}{l}
\dot{z}_{1}(t)=z_{2}(t) \\
\dot{z}_{2}(t)=\dot{z}_{2}(t-1) .
\end{array}\right.
$$

This system is continuously finally observable by the feedback $y=z_{1}(t-1)$ by Theorem 8 .

\section{Conclusion}

We gave some relations between exact null controllability and complete stabilizability of abstract systems in Hilbert spaces. A characterization of complete stabilizability was given for a large class of linear neutral type systems. Necessary conditions of exact null controllability were presented, which conjectured to be also sufficient for neutral type systems, even if they are not in the general case. This also allows the final continuous observability of such systems to be characterized. The following step is to prove the conjecture and to extend such results to the problem of detectability, which is dual to stabilizability.

\section{Acknowledgment}

An earlier version of this work was presented at the International Conference on System Analysis: Modelling and Control, organized in memory of Academician Arkady Kryazhimskiy at the Krasovskii Institute of Mathematics, Ural Branch of the RAS, Ekaterinburg, Russia. The first author wishes to thank Professor Vyacheslav Maksimov and the Organizing Committee of the conference.

\section{References}

Curtain, R.F., Logemann, H., Townley, S. and Zwart, H. (1997). Well-posedness, stabilizability, and admissibility for Pritchard-Salamon systems, Journal of Mathematical Systems, Estimation, and Control 4(4): 493-496.

Curtain, R.F. and Zwart, H. (1995). An Introduction to Infinitedimensional Linear Systems Theory, Texts in Applied Mathematics, Vol. 21, Springer-Verlag, New York, NY.

Douglas, R.G. (1966). On majorization, factorization, and range inclusion of operators on Hilbert space, Proceedings of the American Mathematical Society 17: 413-415. 
Dusser, X. and Rabah, R. (2001). On exponential stabilizability of linear neutral systems, Mathematical Problems in Engineering 7(1): 67-86.

Guo, F., Zhang, Q. and Huang, F. (2003). Well-posedness and admissible stabilizability for Pritchard-Salamon systems, Applied Mathematical Letters 16(1): 65-70.

Hale, J.K. and Verduyn Lunel, S.M. (1993). Introduction to Functional-Differential Equations, Applied Mathematical Sciences, Vol. 99, Springer-Verlag, New York, NY.

Hale, J.K. and Verduyn Lunel, S.M. (2002). Strong stabilization of neutral functional differential equations, IMA Journal of Mathematical Control and Information 19(1-2): 5-23.

Ito, K. and Tarn, T.J. (1985). A linear quadratic optimal control for neutral systems, Nonlinear Analysis 9(7): 699-727.

Khartovskiü, V.E. and Pavlovskaya, A.T. (2013) Complete controllability and controllability for linear autonomous systems of neutral type, Automation and Remote Control 79(5): 769-784.

Kuperman, L.M. and Repin, Ju. M. (1971) On the question of controllability in infinite-dimensional spaces, Doklady Akademii Nauk SSSR 200(3): 767-769, (in Russian). English translation: Soviet MathematicsDoklady 12(5): 1469-1472.

Logemann, H. and Pandolfi, L. (1994) A note on stability and stabilizability of neutral systems, IEEE Transactions on Automatic Control 39(1): 138-143.

Louis, J.-C. and Wexler, D. (1983). On exact controllability in Hilbert spaces, Journal of Differential Equations 49(2): 258-269.

Marčenko, V.M. (1979). On the controllability of zero function of time lag systems, Problems of Control and Information Theory/Problemy Upravlenia $i$ Teoria Informacii 8(5-6): 421-432.

Megan, M. (1975). On the stabilizability and controllability of linear dissipative systems in Hilbert space, Seminarul de Ecuatii Functional, Universitatea din Timisoara 32: 1-15.

Metel' skiı̌, A.V. and Khartovskii, S.A. (2016). Criteria for modal controllability of linear systems of neutral type, Differential Equations 52(11): 1453-1468.

Metel'skiı̌, A.V. and Minyuk, S.A. (2006). A criterion of constructive identifiability and complete controllability of linear time-independent systems of neutral type, Izvestiya Rossiiskoi Akademii Nauk: Teoriya i Sistemy Upravleniya (5): 15-23, (in Russian). English translation: Journal of Computer and Systems Sciences International 45(5): 690-698.

Michiels, W. and Niculescu, S.-I. (2007). Stability and Stabilization of Time-Delay Systems. An Eigenvalue-Based Approach, Advances in Design and Control, Vol. 12, SIAM, Philadelphia, PA.

O'Connor, D.A. and Tarn, T.J. (1983). On stabilization by state feedback for neutral differential equations, IEEE Transactions on Automatic Control 28(5): 615-618.

Olbrot, A.W. and Pandolfi, L. (1988). Null controllability of a class of functional-differential systems, International Journal on Control 47(1): 193-208.
Pandolfi, L. (1976). Stabilization of neutral functional differential equations, Journal of Optimization Theory and Applications 20(2): 191-204.

Pazy, A. (1983). Semigroups of Linear Operators and Applications to Partial Differential Equations, Applied Mathematical Sciences, Vol. 44, Springer-Verlag, New York, NY.

Pritchard, A.J. and Salamon, D. (1987). The linear quadratic control problem for infinite dimensional systems with unbounded input and output operators, SIAM Journal on Control and Optimization 25(1): 121-144.

Rabah, R. and Karrakchou, J. (1997). On exact controllability and complete stabilizability for linear systems in Hilbert spaces, Applied Mathematics Letters 10(1): pp. 35-40.

Rabah, R. and Sklyar, G.M. (2007). The analysis of exact controllability of neutral-type systems by the moment problem approach, SIAM Journal on Control and Optimization 46(6): 2148-2181.

Rabah, R. and Sklyar, G.M. (2016). Exact observability and controllability for linear neutral type systems, Systems and Control Letters 89: 8-15.

Rabah, R., Sklyar, G.M. and Barkhayev, P.Y. (2012). Stability and stabilizability of mixed retarded-neutral type systems, ESAIM Control, Optimization and Calculus of Variations 18(3): 656-692.

Rabah, R., Sklyar, G.M. and Barkhayev, P.Y. (2014). On the exact controllability and observability of neutral type systems, Communications on Mathematical Analysis 17(2): 279-294.

Rabah, R., Sklyar, G.M. and Barkhayev, P.Y. (2016). On exact controllability of neutral time-delay systems, Ukrainian Mathematical Journal 68(6): 800-815.

Rabah, R., Sklyar, G.M. and Rezounenko, A.V. (2005). Stability analysis of neutral type systems in Hilbert space, Journal of Differential Equations 214(2): 391-428.

Rabah, R., Sklyar, G.M. and Rezounenko, A.V. (2008). On strong regular stabilizability for linear neutral type systems, Journal of Differential Equations 245(3): 569-593.

Rhandi, A. (2002). Spectral Theory for Positive Semigroups and Applications, Quaderni di Matematica, Vol. 2, ESE-Salento University Publishing, Lecce.

Richard, J.-P. (2003). Time-delay systems: An overview of some recent advances and open problems, Automatica 39(10): 1667-1694.

Salamon, D. (1983). Neutral functional differential equations and semigroups of operators, in F. Kappel et al. (Eds.), Control Theory for Distributed Parameter Systems and Applications, Lecture Notes in Control and Information Sciences, Vol. 54, Springer, Berlin, pp. 188-207.

Salamon, D. (1984). Control and Observation of Neutral Systems, Research Notes in Mathematics, Vol. 91, Pitman (Advanced Publishing Program), Boston, MA. 
Salamon, D. (1987). Infinite dimensional linear systems with unbounded control and observation: A functional analytical approach, Transactions of American Mathematical Society 300(2): 383-431.

Sklyar, G.M. and Szkibiel, G. (2013). Controlling a nonhomogeneous Timoshenko beam with the aid of the torque, International Journal of Applied Mathematics and Computer Science 23(3): 587-598, DOI: 10.2478/amcs-2013-0044.

Tucsnak, M. and Weiss, G. (2009). Observation and Control for Operator Semigroups, Birkhäuser Advanced Texts, Basel/Birkhäuser, Boston, MA.

Wonham, W.M. (1985). Linear Multivariable Control: A Geometric Approach, 3rd Edn., Springer, New York, NY.

Zabczyk, J. (1976). Complete stabilizability implies exact controllability, Seminarul de Ecuatii Functional, Universitatea din Timisoara 38: 1-7.

Zabczyk, J. (1992). Mathematical Control Theory: An Introduction, Systems \& Control: Foundations \& Applications, Birkhäuser, Boston, MA.

Zeng, Y., Xie, Z. and Guo, F. (2013). On exact controllability and complete stabilizability for linear systems, Applied Mathematical Letters 26(7): 766-768.



Rabah Rabah was a full professor and Directeur de Recherche at École des Mines de Nantes from 1993 up to January 2017. He obtained his Master's and PhD degrees from Kharkov University, Russia. He was an assistant and a full professor in the Department of Mathematics of Oran University (Algeria) from 1981 to 1993 . His main research activity covers systems theory and control at the Research Institute of Communication and Cybernetics, and in collaboration with research teams from France, Morocco, Poland and Ukraine.

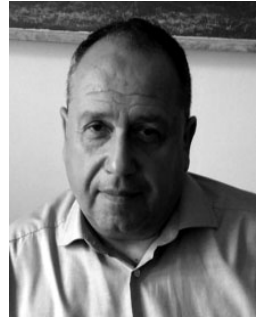

Grigory M. Sklyar has been a full professor at the University of Szczecin since 1997. He obtained his $\mathrm{PhD}$ from Kharkiv University. Currently, he is the head of the Control and Optimization Unit within the Institute of Mathematics. He is an author of over 90 research papers, mainly in system theory and control. He has participated in research projects supported, among others, by DAAD and NATO. He collaborates with researchers from Ukraine (Kharkiv), Germany (Greifswald) and France (Nantes).

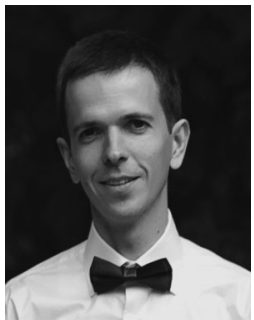

Pavel Yu. Barkhayev is a research fellow at the B. Verkin Institute for Low Temperature Physics and Engineering of the National Academy of Sciences of Ukraine and an associate professor at Karazin Kharkiv National University. He received a Master's degree in 2001 and a PhD degree in 2005 from Karazin Kharkiv National University. His research interests include control theory for dynamic systems, neutral type timedelay systems, and nonlinear finite-dimensional systems of ordinary differential equations.

Received: 16 November 2016 Revised: 30 March 2017 Re-revised: 11 May 2017 Accepted: 7 June 2017 